\title{
Privacy Protection in Bigdata: A Survey
}

\author{
Saumya Gupta ${ }^{a}$, and Dr. Chander Prabha ${ }^{b}$ \\ Research Scholar (CSE), Chandigarh University, Gharuan, India. \\ B Associate Professor (CSE), Chandigarh University, Gharuan, India.
}

Article History: Received: 11 January 2021; Accepted: 27 February 2021; Published online: 5 April 2021

\begin{abstract}
Bigdata becomes a significant sector and academics research topic. Bigdata is a two-edged sword. The rising volume of information together will increase the likelihood of blundering non-public data privacy. Due to many new technologies and innovations that pervade our everyday lives, like smartphones and social networking apps, and the Internet of Things-based intelligent-world systems, the large amount of data generated in our world has exploded. During this data processing, storage, and the use of the information it can quickly cause personal information exposure and the difficulty of interpreting the information. The aim is to incorporate this range of information into one framework for big data management and to recognize problems regarding privacy. This paper begins with the introduction of bigdata, its process, protection issues, and tools which are used to solve its problems.
\end{abstract}

Keywords: Big data, security, protection, and privacy

\section{Introduction}

Bigdata relates to the bulk of data that came from dissimilar data originators and have, unlike formats. Even before, large information had been collected in databases, but due to the assorted nature of such data, conventional related database structures are unable to handle this data. Bigdata is more than just a cluster of databases of various formats; this is a significant asset that could be used to achieve an uncountable advantage [1]. The definition of bigdata is investing in business intelligence capability, profitable intelligence, improved perspective, and higher cognitive process. In the digital era, continuous increase of data is captured from IoT (internet-of-things) such as RIFD (radio-frequency-identification), remote sensing, software logs, microphones, wireless sensor network, and many more, which generate large and complex data set in huge volume, the information could be organized, semiorganized and unstructured for that customary applications for information preparing are insufficient [2] [11]. With the quick development of the Internet, the crime rate increases rapidly. Hackers can easily get information about our activities and lead to illegal activities.

In [4], this concept is referred to as Bigdata "youth of today of techniques and structures aimed at generating, investigating, and analyzing heavy-volume information from very broad quantities of data". This definition illustrates the $3 \mathrm{~V}$-volume, velocity, and variety characteristics or template of big data. IBM defined Bigdata using $4 \mathrm{~V}$ 's characteristics by adding 'veracity' in it. In 2014, a new characteristic included 'value' to extend the model to $5 \mathrm{~V}$ 's, characteristics as shown in figure 1.

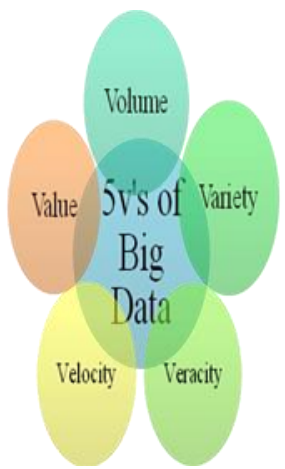

FIGURE 1: 5V'S OF BIGDATA

The amount of data produced by emails, Twitter messages, videos, video clipper is generated in speed 'Velocity', social media, mobile phones, vehicles, credit card, M2M sensors, image data generated in 'Volume' every microsecond, and data generated from social media sites, e-mails, web pages, papers, sensor devices, and weblogs files, are generated in unstructured 'Variety'. Table 1 elaborates on all 5V's. Some other characteristics of bigdata are exhaustive, fine-grained, and uniquely lexical, relational, extensional, scalability, and variability. 
- In complexity, linking, mixing, cleaning, and transforming data through applications from diverse sources is an activity. Connecting and correlating associations, power structures, and different data links is also important, and data may escalate rapidly.

- In scalability, if the data volume will increase easily.

- In extensional, whether new areas can also be easily integrated or modified in each component of the data gathering.

- In relational, if the collected data includes collective areas that might allow different databases to be merged or meta-analyzed.

Various methods have been established in recent years to ensure the confidentiality of bigdata. Depending on the bigdata life cycle stages, i.e. information generation, storage, and processing, these processes can be grouped. For privacy protection, access limitations just as adulterating information procedures are utilized in the information generation process [10].

\begin{tabular}{|c|c|c|c|c|c|}
\hline & Volume & Variety & Velocity & Veracity & Value \\
\hline Definition & $\begin{array}{l}\text { It is the } \\
\text { operation that } \\
\text { the incredible } \\
\text { amount of data } \\
\text { generated or } \\
\text { stored } \\
\text { information. }\end{array}$ & $\begin{array}{l}\text { Refers to data } b \\
\text { eing generated } i \\
\text { s not a single ca } \\
\text { tegory, and the } \\
\text { data systems ar } \\
\text { e complex. }\end{array}$ & $\begin{array}{l}\text { This refers to } \\
\text { the rate of } \\
\text { producing, } \\
\text { processing, and } \\
\text { analyzing huge } \\
\text { amounts of } \\
\text { data. }\end{array}$ & $\begin{array}{l}\text { It is the operatio } \\
\mathrm{n} \text { that determine } \\
\mathrm{s} \text { the reliability a } \\
\mathrm{nd} \text { trust of the in } \\
\text { formation used i } \\
\mathrm{n} \text { the decision- } \\
\text { making process. }\end{array}$ & $\begin{array}{l}\text { Refers to the } \\
\text { importance of } \\
\text { the study of } \\
\text { BigData and } \\
\text { data's economic } \\
\text { value. }\end{array}$ \\
\hline Sources & $\begin{array}{l}\text { Petabytes } \\
\text { Tables/files } \\
\text { Records } \\
\text { Transactions }\end{array}$ & $\begin{array}{l}\text { Structured } \\
\text { Semi-structured } \\
\text { Unstructured } \\
\text { All the above }\end{array}$ & $\begin{array}{l}\text { Batch } \\
\text { Streams } \\
\text { Real-time } \\
\text { Near-time }\end{array}$ & $\begin{array}{l}\text { Trustworthiness } \\
\text { Availability } \\
\text { Accountability } \\
\text { Authenticity } \\
\text { Reputation }\end{array}$ & $\begin{array}{l}\text { Structured } \\
\text { Semi-structured } \\
\text { Unstructured } \\
\text { All the above }\end{array}$ \\
\hline
\end{tabular}

Table 1 Illustrate 5V's of Bigdata [12]

\section{Related Work}

In this paper [1], Dittrich, J., \& Quiané-Ruiz, J. A. (2012) discuss, many Hadoop MapReduce analysis methods can be utilized to improve the presentation by an equivalent amount. We demonstrate these methods throughout this review. Next, we're going to get the community acquainted with Hadoop MapReduce briefly and inspire their use for bigdata storage.

In this paper [2], Acharjya, D. P., Ahmed, K. (2016) said that this survey provides a platform for multiple stages to discuss big data. It also creates a whole new frontier with scientists to create an alternative based on global science questions as well as problems. Some are planned for cluster handle, while some are good for real-time analysis. Every bigdata system also has its unique features.

In this paper [3], Altman, Micah, Alexandra B. Wood, David O'Brien, and Urs Gasser (2018), examine a variety of semi-permanent analytical studies to identify the attributes driving the distinctive danger and edge sets and, therefore, the procedures maintained to protect analysis knowledge subject areas from lengthy-term data protection risks, they find that many significant data operations in public and private sector environments include similar characteristics and threats to long-term analysis but are subject to less monitoring and regulation. They presume that some way or another the dangers presented by huge information after some time can all the more likely be deciphered as a part of time factors like age, length, and recurrence and non-worldly factors, for example, segment thickness, standard deviation, measurement, and anticipated exploratory use.

In this paper [4], Meh-mood, Abid, Iyn-karan Natgunanathan, Yong-Xiang, Guang-Hua, and Song-Guo (2016), provide a comprehensive survey of the BigData Privacy Framework and to present challenges to existing frameworks. They performed a significant study on privacy problems when dealing with complex data. The paper, in general, highlights the architecture of bigdata and also the processes of privacy protection at each point of the bigdata life cycle. Besides, they are addressing the issues and future directions of research related to the protection of privacy in bigdata.

In this paper [5], Hashem, Ibrahim, Ibrar, Nor Badrul Anuar, Salimah, Abdullah, and Samee (2015), in this thesis they explore the emergence of massive data in cloud computing. They suggested a description of bigdata, a theoretical understanding of bigdata, and a framework of cloud providers. This framework was related to many generic cloud data systems. Also addressed is the collaboration between big data and cloud services, bigdata processing, and Hadoop technologies. Recent MapReduce activities and related technology have been described. They already examined many of the problems throughout the processing of big data.

In this paper [8], Sangeetha, S., and G. Sudha Sadasivam (2019) provide a brief review of the multiple data processing methods protecting the confidentiality and their use in the BigData era, the paper features the territory 
of - the-workmanship assurance of information gathering structures and suggestions for the utilization of such systems to the bigdata condition. Knowledge of the essence of big data and its technical equivalents is presented. Huge information pressure calculations, for example, Hadoop and flash help specialists address industry obstructions by furnishing business insight arrangements with dashboards, reports, inquiries, and prescient investigation, for example, streamlining, and anticipating factual examination.

In this paper [9], Katal, Avita, Mohammad Wazid, and R. H. Goudar (2013), discuss Bigdata technologies and their significance in the contemporary world as well as current initiatives that are successful and significant in turning the materialistic worldview into broad science and technology as well. Also explained in detail are the numerous difficulties and concerns in integrating and embracing Bigdata Technology, its resources (Hadoop) together with the challenges faced by Hadoop. The paper ends with the application of Better Bigdata to adopt.

In this paper [10], Priyank, Manasi Gyan, and Nilay Khare (2016). they aimed to provide a comprehensive review of the frameworks through protecting the privacy of big data and to present a problem to established methods. This paper also discusses the new privacy and security protection strategies in big data such as trapping a way around the problem, ethnicity-based multifactor authentication, comparative confidentiality, privacy that protects bigdata publication, and the rapid asymmetric encryption of bigdata flows.

In this article [14], Hu, H., Wen, Y., Chua, T.S. and Li, X (2014), introduce a research review and framework overview for Bigdata Analytics systems, aimed at providing non-expert audiences with a big picture and building a do because it-yourself mentality for experienced readers to develop their Bigdata approaches. Next, they address the concept of big data or examine the complexities of bigdata. First, they provide a structured model for breaking down big data frameworks into four hierarchical components, i.e. information creation, data acquisition, data management, and data analysis. Such four components create a network with large data quality.

In this paper [17], Liang, F., Yu, W., An, D., Yang, Q., Fu, X., \& Zhao, W. (2018), the main objective of this research is to have a transparent and in-depth intelligence of big data trade. They discussed the scope of issues domain-specific processing, data sharing, and data security, and outlined fields that remain unanswered in an attempt to somehow encourage the technology development of big data.

\section{Mechanisms of Bigdata}

In this section, we will discuss mechanisms of bigdata, which can be grouped based on stages of bigdata lifecycle [4]. During its life cycle, big data must go through multiple phases, as shown in Fig 2. Information is already being dispersed, and new systems have been built to hold and manage large amounts of information databases.

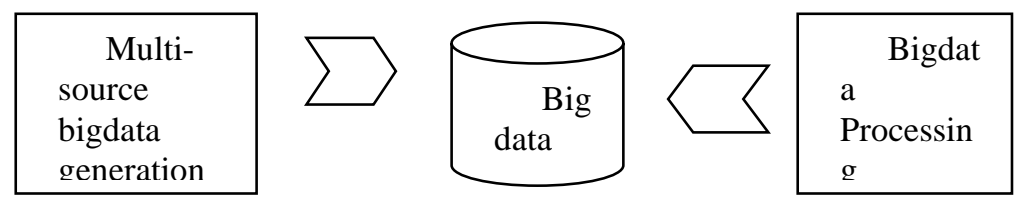

Figure 2 Illustrate bigdata life cycle

These processes can be categorized depending on the stages of bigdata life cycle, that is, information generation storage, and processing as shown in fig 3.

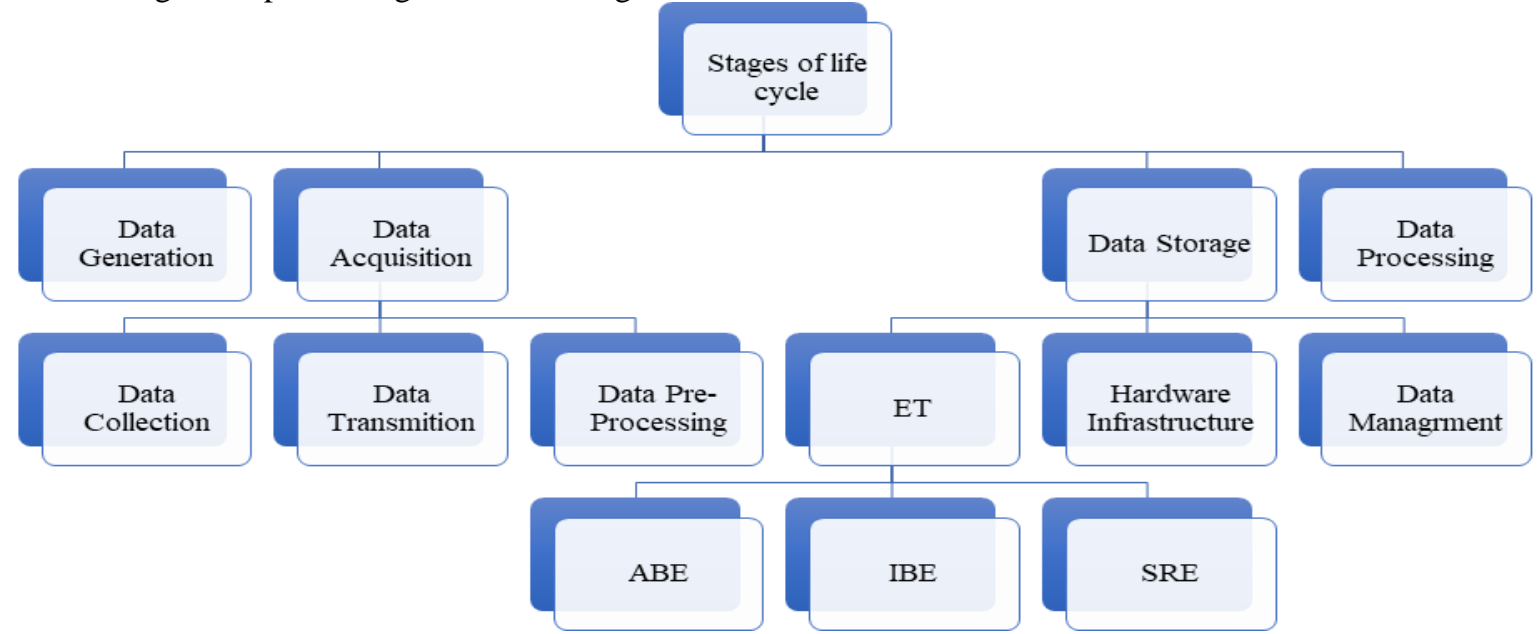

Figure 3 Stages of bigdata life cycle [4].

For privacy protection, access limitation, and falsification of information techniques are used in the data generation phase [16]. While access restriction techniques (ART) are intended for limiting access to private information for individuals, verifying data techniques change the actual data before another untrustworthy community is published.

- Information (Data) generation, data from various decentralized resources can be produced. Over the past several years, the volume of data produced by people and computers has grown. Its trend is characterized by its 
generation rate [17]. Especially, we loosely describe information generation behaviors in three consecutive phases: organized, semi-organized, or unstructured data.

- Information (Data) acquisition, the assignment of the information procurement stage is to total data in an advanced structure for additional capacity and examination. Instinctively, the securing procedure comprises of three sub-steps, information assortment, information transmission, and information pre-preparing. There is no exacting request between information transmission and information pre-preparing; consequently, information prehandling activities can happen before information transmission as well as after information transmission. Its stages consist of three subtasks:

Information (data) Collection: Information assortment alludes to the way toward recovering crude information from certifiable items. The procedure should be very much planned. Something else, off base information assortment, would affect the ensuing information investigation method and at last lead to invalid outcomes. Simultaneously, information assortment strategies not just rely upon the material science attributes of information sources yet also the goals of information examination.

Information (data) Transmission: we accumulate the crude information; we should move it into an information stockpiling framework, ordinarily in a server farm, for resulting handling. The transmission technique can be partitioned into two phases, IP spine transmission, and server farm transmission.

Information (data) Pre-processing: the gathered informational indexes may have various degrees of value as far as clamor, repetition, consistency, and so on. Transferring and storing raw data would have the necessary costs. On the interesting side, certain information investigation strategies and applications may have severe necessities on information quality. All things considered, information pre-preparing procedures that are intended to improve information quality ought to be set up in huge information frameworks.

- In the data storage process, they are mainly based on encryption techniques (ET) [4]. It is also possible to separate encryption-based techniques (EBT) into attribute-based encryption (ABE), identity-based encryption (IBE), and storage route encryption (SRE). Therefore, hybrid clouds are used to protect sensitive information as confidential data is stored in private clouds. Such processes can also be categorized through strategies focused on segmentation, identification, and relationship rule processing. Although segmentation and identification divide the incoming data into separate groups, the valuable connections and patterns in the incoming data are found in association rule processing [10].

There are two aspects of a data storage device, i.e., as shown in fig 3, hardware infrastructure, and data management [14].

$>$ Hardware infrastructure relates to the use of ICT tools for different tasks (such as compute clusters).

$>$ Data management relates to the collection of applications implemented to handle and search massivescale datasets in addition to that of the hardware infrastructure. It could also have multiple APIs to communicate with data stored and evaluate these.

- The data processing, stage generally serves the purpose of gathering, transmitting data, immediate preprocessing, and collecting valuable information. The collection of information is important since information can come through multiple sources, i.e. pages containing text, images, and videos [4].

Processing of data [16] involves the privacy of data preservation (PPDP) and the extraction of data knowledge. To protect data privacy, PPDP uses anonymization techniques such as generalization and denial. Securing the utility of the information while ensuring privacy is a major challenge for PPDP.

\section{Bigdata Challenges}

- $\quad$ Privacy: Data protection is the right of some power over the gathering and the use of personal details. Data protection is the right of a person or group of people and prevents the use of personal data to individuals apart from those to which it is given. The discovery of personal information during internet transmission is a serious issue for user privacy [10]. Suppose that the company and customized product collect the data but unknowingly infringe people's privacy [13].

- Security: Security is the norm of using the software, procedures, and training to protect data and information resources from- unauthorized access, disclosure, disturbance, alteration, inspection, recording, and destruction [10]. Look at healthcare information security, healthcare organizations store, manage and distribute vast amounts of data to enable secure and proper care delivery, the weakness is the lack of professional assistance and the lack of safety.

- $\quad$ Lack of creativity for privacy protection: In the era of bigdata, information is disseminated at an extremely fast pace. At the same time as the transmission of information is not of high value due to inadequate data information control, lack of technical support, poor monitoring process, and information loss vulnerability. The cost of itself will have many adverse and detrimental effects on people, firms, and even culture, leading to greater economic losses [7].

- Risks for data security: This kind of network environment has become increasingly important for the security of mobile data with intelligent data terminals since the advent of the bigdata era and the exponential growth of the Internet. Current China has become the largest smart mobile terminal market in the world. Not only do these large numbers of mobile terminals eat up people's energy and time, but they also store more in-house personal data. 
People are currently having serious problems with bigdata protection, and they think bigdata is not secure. Not only the difficulty of bigdata [7].

- The main threat of data collection: In the observational study, an analysis of data protection threats shows that the current three time-related data attributes raise identity threat [3]:

Age: Age is described as the period between existing data gathering and its evaluation. Data can be analyzed immediately since complete set, just like in the scenario of a mobile phone app targeting user-based advertising, or data can be analyzed decades forward to set, along with public documents that have been safeguarded from transparency for several years.

Period: Time frame refers to the period duration in which these items are evaluated frequently. Many data surveys make predictions at a specific moment in time, including a bridge-sectional research study, whereas others participate in a collection for years or even centuries, such as with a deep-term statistical survey or a lengthystanding networking site.

Frequency: Frequency or duration of physical actions within the same issue. Large-frequency data gathering sources involve electronic fitness applications and gadgets which monitor datasets like position and pulse rate constantly. Many medical studies, just from the other side of the scale, can gather information from researchers once a year, only once every few decades.

- $\quad$ Resistance from the company: It's not just the technical dimensions of big data that can also be daunting - citizens can become a challenge as well. When questioned regarding the current barriers to this change of society, participants referred to three major hurdles inside the institutions:

$>$ Extremely limited institutional cohesion (4.6\%)

$>$ Loss of level management $(41.0 \%)$

$>$ Market reluctance and lack of knowledge $(41.0 \%)$

We would have to do some activities better for corporations to focus exclusively on both the benefits provided by bigdata. As well as for large institutions this kind of transition can be immensely difficult.

\section{Tools of Bigdata Privacy}

This paragraph explains the strategies that can be applied to privacy-preserving information (data) mining (PPDM) in bigdata. In data processing, privacy protection is generally classified as follows (as shown in Fig 4); nevertheless, the following hierarchy is not restricted [8]:

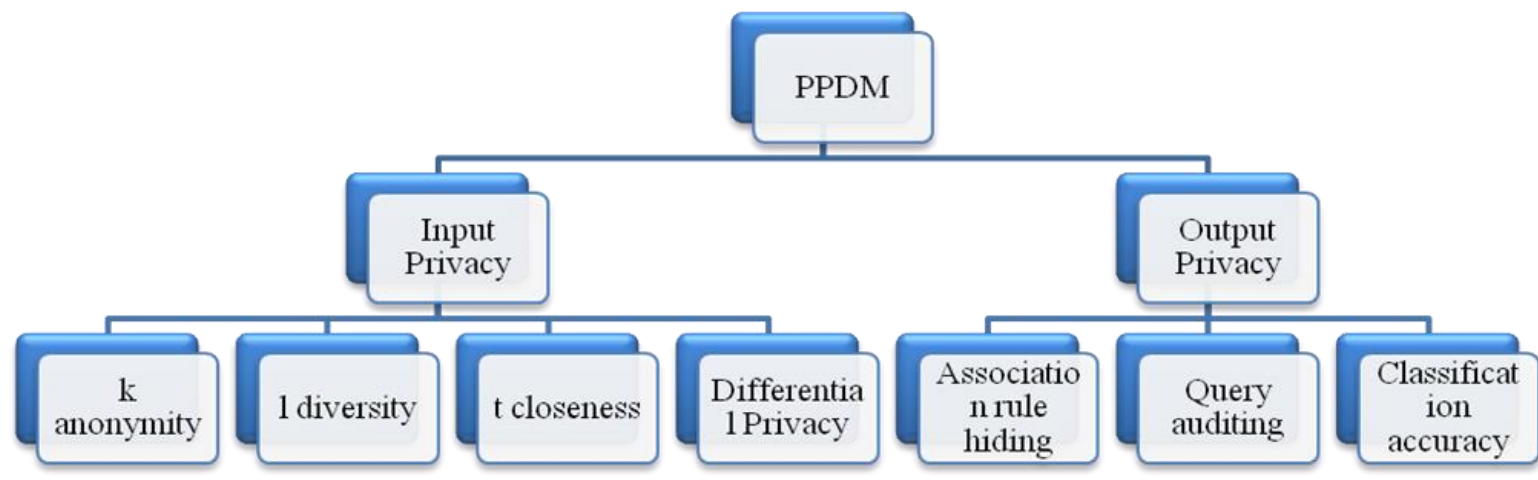

Figure 4 PPDM hierarchy

Throughout input privacy, the release of anonymized data is primarily concerned. The outcome is interrupted or audited throughout production privacy to preserve security. There are also methods focused on cryptography other than these specific classifications.

Some current methods for protecting the confidentiality of bigdata are outlined below [10]:

De-identifying: De-identification is a standard data analysis strategy in which information should also be purified with generality (removing sort of semi-identifiers with the less precise yet semi-consistent values) and elimination (not revealing those principles of any kind) until launching for data collection to preserve personal privacy. There will be three types for de-identification-privacy-preserving, respectively, K-anonymity, L-diversity, and Tcloseness.

$>$ K-anonymity: For releasing meta tags, the k-anonymity confidentiality criterion needs where each correlation classification contains $\mathrm{k}$ information [8].

$>$ L-diversity: A correlation group was said to have 1-diversity whether the critical variable has different values in at minimum 1 "excellently-represented" [8].

$>$ T-closeness: If the difference between the representation of the specific component throughout this category and also the distributions of the component throughout the entire table isn't much of a minimum $\mathrm{t}$, a correlation group is shown to have t-closeness [8].

$>$ Differential privacy: Differential Privacy is now a technique that offers investigators, server developers an opportunity to receive valuable information through repositories comprising personally identifiable 
information without exposing the individual's identity. It is achieved by adding limited disturbance in the server process content [10].

Output Privacy: The key accentuation of yield protection is to irritate the yield of different information mining calculations to guarantee security. A portion of the techniques is examined in the accompanying segment.

$>$ Association rule hiding: An affiliation (association) rule is delicate if they help and certainty of the standard are more prominent than a base limit. Backing and certainty are the essential proportions of some random affiliation rules.

$>$ Query auditing: Inquiry inspecting is a security safeguarding component to explore and maintain a strategic distance from private information exposure from the database. The examination is done both on the web and disconnected modes. In online mode the meeting is intuitive and in disconnected mode, the inquiry reaction occurs at the break stretches. They look at total honesty and halfway revelation of the database.

$>$ Classification accuracy: Choice (decision) tree arrangement is a significant information mining strategy to build up a characterization framework. Insecurity saving information mining, the test is to build up the choice tree from irritated information which gives a novel recreation method that intently approximates the first appropriation.

\section{Conclusion}

The advent of the Bigdata revolution has not only created significant social change opportunities but has also brought various challenges to the protection of information to society, making it a priority to protect the privacy of personal data. To ensure the security and privacy of bigdata information, it is not only important to have a large number of professional private information security technologies, but also to raise awareness of citizens ' privacy in our country to enforce data security [7]. In the present, therefore, different approaches for protecting the privacy of mining can be researched and applied. As just that, there seems to be considerable room for further work into bigdata methods for protecting privacy.

\section{References}

1. Acharjya, D. P., \& Ahmed, K. (2016). A survey on big data analytics: challenges, open research issues, and tools. International Journal of Advanced Computer Science and Applications, 7(2), 511-518.

2. Altman, Micah, Alexandra B. Wood, David O'Brien, and Urs Gasser. "Practical approaches to big data privacy over time." (2018).

3. Bayardo, Roberto J., and Rakesh Agrawal. "Data privacy through optimal k-anonymization." In 21st International conference on data engineering (ICDE'05), pp. 217-228. IEEE, 2005.

4. Dittrich, J., \& Quiané-Ruiz, J. A. (2012). Efficient bigdata processing in Hadoop MapReduce. Proceedings of the VLDB Endowment, 5(12), 2014-2015.

5. Hashem, Ibrahim Abaker Targio, Ibrar Yaqoob, Nor Badrul Anuar, Salimah Mokhtar, Abdullah Gani, and Samee Ullah Khan. "The rise of "big data" on cloud computing: Review and open research issues." Information systems 47 (2015): 98-115.

6. Hassan, Mohammed K., Ali I. El Desouky, Sally M. Elghamrawy, and Amany M. Sarhan. "Big Data Challenges and Opportunities in Healthcare Informatics and Smart Hospitals." In Security in Smart Cities: Models, Applications, and Challenges, pp. 3-26. Springer, Cham, 2019.

7. Https://en.wikipedia.org/wiki/Big_data\#Architecture

8. Hu, H., Wen, Y., Chua, T.S., and Li, X., 2014. Toward scalable systems for big data analytics: A technology tutorial. IEEE Access, 2, pp.652-687.

9. Jain, Priyank, Manasi Gyanchandani, and Nilay Khare. "Big data privacy: a technological perspective and review." Journal of Big Data 3, no. 1 (2016): 25.

10. Katal, Avita, Mohammad Wazid, and R. H. Goudar. "Big data: issues, challenges, tools, and good practices." In 2013 Sixth international conference on contemporary computing (IC3), pp. 404-409. IEEE, 2013.

11. Liang, F., Yu, W., An, D., Yang, Q., Fu, X., \& Zhao, W. (2018). A survey on big data market: Pricing, trading, and protection. IEEE Access, 6, 15132-15154.

12. Matturdi, Bardi, Xianwei Zhou, Shuai Li, and Fuhong Lin. "Big Data security and privacy: A review." China Communications 11, no. 14 (2014): 135-145.

13. Mehmood, Abid, Iynkaran Natgunanathan, Yong Xiang, Guang Hua, and Song Guo. "Protection of big data privacy." IEEE Access 4 (2016): 1821-1834

14. Puri, Ganesh D., and D. Haritha. "Survey big data analytics, applications, and privacy concerns." Indian Journal of Science and Technology 9 (2016): 1-8.

15. Sangeetha, S., and G. Sudha Sadasivam. "Privacy of Big Data: A Review." In Handbook of Big Data and IoT Security, pp. 5-23. Springer, Cham, 2019.

16. Torra, Vicenç, Guillermo Navarro-Arribas, and Klara Stokes. "Data privacy." Data Science in Practice. Springer, Cham, 2019. 121-132.

17. Zhang, Dongpo. "Big data security and privacy protection." In the 8th International Conference on Management and Computer Science (ICMCS 2018). Atlantis Press, 2018. 